\title{
Remarks on cannibalism and pattern formation in spatially extended prey-predator systems
}

Stefano Fasani

Sergio Rinaldi (rinaldi@iiasa.ac.at)

\section{Approved by}

Ulf Dieckmann

Director, Evolution and Ecology Program

February 2015 Institute, its National Member Organizations, or other organizations supporting the work. 


\title{
Remarks on cannibalism and pattern formation in spatially extended prey-predator systems
}

\author{
Stefano Fasani ${ }^{1}$, Sergio Rinaldi ${ }^{1-3}$
}

${ }^{1}$ DEI, Politecnico di Milano

Via Ponzio 34/5, 20133 Milano, Italy
${ }^{2}$ Evolution and Ecology Program, International Institute for Applied Systems Analysis 2361 Laxenburg, Austria
${ }^{3}$ To whom correspondence should be addressed Ph: +39 022399 3563; Fax: +39 0223993412 rinaldi@elet.polimi.it




\section{Abstract}

The relationships between cannibalism and pattern formation in spatially extended preypredator systems are studied with a model that degenerates, in the absence of cannibalism, into the most standard prey-predator model, known as Rosenzweig-MacArthur model. The analysis is based on the theory developed long ago by Turing in his famous paper on morphogenesis, but in a special form which allows one to decouple the role of demographic parameters from that of diffusive dispersal. The proofs are given in terms of prey and predator nullclines because ecologists are mainly familiar with this technique. The final result of the analysis is that spatial pattern can exist only in systems with highly cannibalistic and highly dispersing predator provided the attractor of the system in the absence of cannibalism is a limit cycle. This result is more simple and more complete than that published in this journal a few years ago by Sun and coauthors.

Key words: prey-predator models, spatial pattern, Turing instability, cannibalism, dispersal. 


\section{Introduction}

Pattern formation in spatially extended prey-predator systems has been studied in ecology for more than thirty years $[6,4,22,23,1,12,2,11,5,17,7,13,14,18,20]$. These investigations, based on the notion of diffusive instability and on the results obtained by Turing [21], show that simple prey-predator interactions can be responsible of patchiness in perfectly homogeneous environments.

Even if cannibalism is a widespread phenomenon among a variety of taxa [10], only one paper (published in this journal) has been devoted to the relationship between cannibalism and pattern formation [20]. However, the analysis of Sun and coauthors is not complete, though accompanied by numerous and interesting simulations, and is performed with a model which is realistic only for very weak cannibalism. For these reasons, we present in this paper a complete analysis of the problem obtained with a biologically sound model of cannibalism [9]. Moreover, we use the recently proposed notion of potential Turing instability [8], which allows one to decouple the role played by demographic factors from that of dispersal of the populations involved.

The results we obtain are general and can be summarized with the following two simple statements :

(i) If a system has a stable equilibrium when the predator in non-cannibalistic, the introduction of any degree of predator cannibalism can not promote pattern formation.

(ii) The addition of cannibalism in a system that has a stable limit cycle when the predator is non-cannibalistc promotes spatial pattern provided predator cannibalism and dispersal are sufficiently high. 


\section{The model}

Spatially extended prey-predator systems can be described, under the standard assumption of diffusive dispersal, by a PDE of the form

$$
\begin{aligned}
& \frac{d x}{d t}=f(x, y, p) x+d_{x} \nabla^{2} x \\
& \frac{d y}{d t}=g(x, y, p) y+d_{y} \nabla^{2} y
\end{aligned}
$$

where $x$ and $y$ are prey and predator biomass depending upon time and space in a given domain of $\mathbb{R}^{2}, f$ and $g$ are per-capita growth rates depending upon demographic parameters $p$, and $d_{x}$ and $d_{y}$ are the non-negative dispersal coefficients of the two populations. Typically, in order to have a well posed problem, zero-flux or periodic boundary conditions are also imposed.

A positive, homogeneous and stationary solution $(\bar{x}, \bar{y})$ of $(1)$ (characterized by $f=g=$ 0 ) can be stable in absence of diffusion (i.e. for $d_{x}=d_{y}=0$ ) but unstable for suitable pairs $\left(d_{x}, d_{y}\right)$. In other words, an equilibrium $(\bar{x}, \bar{y})$ can be stable in a lumped prey-predator model

$$
\begin{aligned}
& \frac{d x}{d t}=f(x, y, p) x \\
& \frac{d y}{d t}=g(x, y, p) y
\end{aligned}
$$

but unstable in its spatially extended version (1). This somehow counterintuitive phenomenon, first investigated by Turing in a celebrated paper [21], is known as diffusion-induced instability, but is also called Turing instability.

The key result of Turing analysis (see also [19]) is that diffusion induced instability is equivalent to the instability of the matrix

$$
C=J(p)-D
$$


where $J(p)$ is the Jacobian of $(2)$ at the equilibrium $(\bar{x}, \bar{y})$, i.e.

$$
J(p)=\left[\begin{array}{ll}
\bar{x} \frac{d f}{d x} & \bar{x} \frac{d f}{d y} \\
\bar{y} \frac{d g}{d x} & \bar{y} \frac{d g}{d y}
\end{array}\right]
$$

and $D$ is the diagonal matrix with $d_{x}$ and $d_{y}$ as diagonal elements. If $d_{x}=d_{y}$, i.e. if $D$ is proportional to the identity matrix, the spectrum of $C$ in (3) is simply the spectrum of $J$ shifted to the left, so that $C$ can not be unstable if $J$ is stable. This is why the dispersion coefficients must be unbalanced in order to have Turing instability.

As shown in $[19,8]$, the problem of finding triples $\left(p, d_{x}, d_{y}\right)$ for which $(\bar{x}, \bar{y})$ is stable in (2) but unstable in (1) can be solved in two steps, namely :

(i) find values of $p$ for which one diagonal element of the Jacobian matrix $J$ is positive. Notice that if $J_{i i}>0$ (i.e. if $i$ is the so-called activator) then $J_{j j}<0$ since $\operatorname{tr}(J)<0$.

(ii) determine (if needed) the dispersal coefficients $\left(d_{x}, d_{y}\right)$ realizing Turing instability by imposing that $C$ is unstable. This is always possible if the activator disperses sufficiently less than the other population (inhibitor).

This decomposition, which has never been systematically exploited in the literature, greatly simplifies the analysis, in particular when the identification of the factors promoting or inhibiting diffusion-induced instability is the problem of concern.

The most standard prey-predator model [16] is composed of a logistic prey and a type II predator and is therefore described by

$$
\begin{aligned}
& \frac{d x}{d t}=\left[r\left(1-\frac{x}{K}\right)-\frac{a y}{1+a h x}\right] x \\
& \frac{d y}{d t}=\left[e \frac{a x}{1+a h x}-d\right] y
\end{aligned}
$$

where $r$ and $K$ are prey net growth rate and carrying capacity and $a, h, e$ and $d$ are predator 


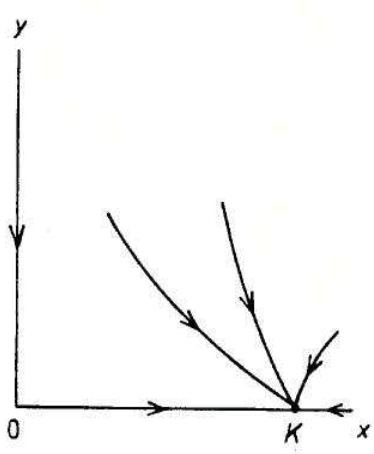

(a)

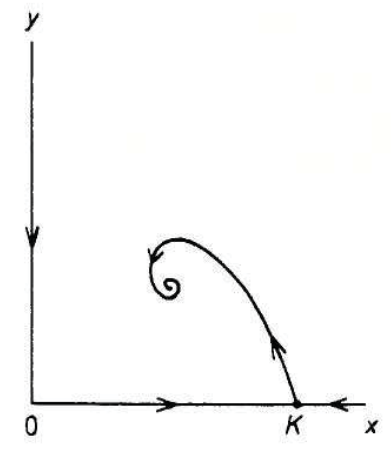

(b)

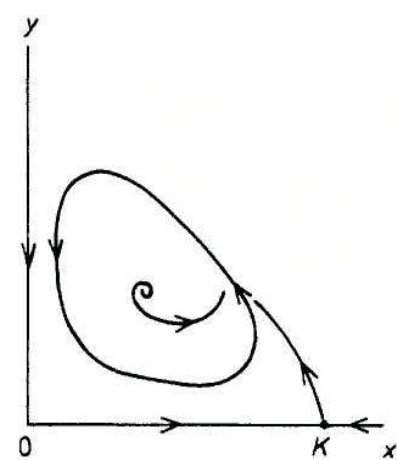

(c)

Figure 1: Phase portraits of system (4): (a) predator extinction, (b) stationary coexistence and (c) cyclic coexistence

attack rate, handling time, efficiency and death rate, respectively. Model (4) is of the form (2) and has three possible state portraits [15] as shown in Fig.1. If the product of attack rate and carrying capacity is low, i.e.

$$
a K<d /(e-d h)
$$

(notice that $e>d h$ because it is reasonable to assume that predator invade when the food available to them is very abundant), then predator die-off in the long term (Fig.1a), while the system tends toward a positive attractor if the opposite inequality is satisfied. More precisely, if

$$
d /(e-d h)<a K<\left(\frac{e}{h}+d\right) /(e-d h)
$$

the system tends toward a stable positive equilibrium $(\bar{x}, \bar{y})$ (Fig.1b) while if

$$
a K>\left(\frac{e}{h}+d\right) /(e-d h)
$$

the system tends toward a stable limit cycle (Fig.1c).

Since the per-capita growth rate $g$ does not depend upon predator density, the second diagonal element of the Jacobian $J$ in $(3)$ is zero, i.e. $J_{22}=0$ for all parameter values, while 
$J_{11}<0$ at the positive equilibrium $(\bar{x}, \bar{y})$ of Fig. 1 since $\operatorname{tr}(J)<0$ at a stable equilibrium. This means that Turing condition $\left(J_{11} J_{22}<0\right)$ can not be satisfied in model $(4)$, although it is almost satisfied since $J_{11} J_{22}=0$. In other words, model (4) is critical in the context of Turing instability. This has been explicitly recognized by Sun et al. [20] as well as by Alonso et al. [1].

In order to study the relationship between cannibalism and pattern formation we must modify model (4) by taking into account that a cannibalistic predator has different attack rates $(a$ and $A$ ) and handling times $(h$ and $H$ ) for the prey and for the predator, as well as two different efficiencies ( $e$ and $E$ ) in transforming predated units into newly born predator. The corresponding model is [9]:

$$
\begin{aligned}
& \frac{d x}{d t}=\left[r\left(1-\frac{x}{K}\right)-\frac{a y}{1+a h x+A H y}\right] x \\
& \frac{d y}{d t}=\left[e \frac{a x}{1+a h x+A H y}+E \frac{A y}{1+a h x+A H y}-\frac{A y}{1+a h x+A H y}-d\right] y
\end{aligned}
$$

where $E<1$ because the biomass of cannibalized predator is greater than the corresponding biomass of newly born predator. Thus, in conclusion, the model with cannibalistic predator can be given the form

$$
\begin{aligned}
& \frac{d x}{d t}=\left[r\left(1-\frac{x}{K}\right)-\frac{a y}{1+a h x+A H y}\right] x \\
& \frac{d y}{d t}=\left[\frac{a e x-A(1-E) y}{1+a h x+A H y}-d\right] y
\end{aligned}
$$

Since model (7) degenerates into model (4) when $A$ tends to zero, the cannibalistic attack rate $A$, from now on simply called cannibalism, is the most natural control parameter for our discussion.

It is worth noticing that the model used by Sun et al. in [20] (see their eq.7) is different from our model (7) and can, at most, be considered as a sort of approximated model valid 


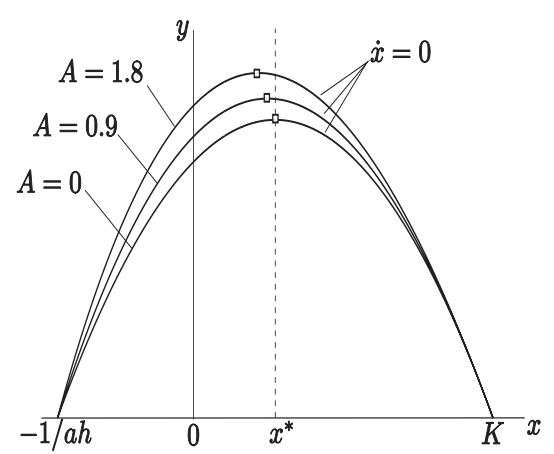

(a)

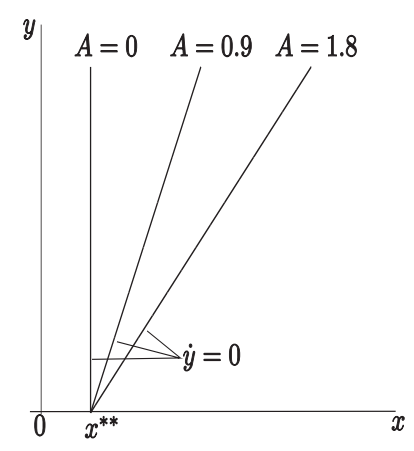

(b)

Figure 2: Prey (a) and predator (b) nontrivial isoclines for different values of cannibalism $(A)$. The figures are obtained with the set of parameters $r=0.5, K=5, a=2, H=0.4, h=0.22, e=0.5, E=0.4$ and $d=0.7$.

for small values of cannibalism: as such, it can not be used to support any conclusion on pattern formation in systems with high cannibalism.

\section{Analysis of the model}

In this section we prove the two properties reported in the Introduction, which can now be more precisely formulated as follows.

(i) If condition (5) is satisfied, predator cannibalism $(A)$ can not promote pattern formation in model $(1,4)$.

(ii) If condition (6) is satisfied, spatial pattern can emerge in model $(1,4)$, provided predator cannibalism $(A)$ and dispersal $\left(d_{y}\right)$ are sufficiently high.

The non-trivial prey nullcline $d x / d t=0$ for model (4) is

$$
y=\frac{r(1-x / K)(1+a h x)}{a-r(1-x / K) A H}
$$

It is a unimodal curve intersecting the $x$-axis at two points $(x=-1 / a h$ and $x=K)$ that do not depend on canibalism $(A)$. By contrast, for all values of $x$ in the range $(-1 /(a h), K)$, 
any increase of $A$ gives rise to an increase of $y$, as shown in Fig.2a. Moreover, after some algebra one obtains

$$
\frac{d y}{d x}=\frac{-\frac{a}{K}(1+a h x)+a\left(1-\frac{x}{K}\right) a h-r a h A H\left(1-\frac{x}{K}\right)^{2}}{\left(a-r\left(1-\frac{x}{K}\right) A H\right)^{2}}
$$

which allows one to check that $d y / d x$ is negative at the central point

$$
x^{*}=\frac{K-\frac{1}{a h}}{2}
$$

of the interval $[-1 / a h, K]$, where the nullcline corresponding to $A=0$ (the RosenzweigMacArthur parabola) has its maximum. In fact, for $x=x^{*}$ the first two terms at the numerator of (8) cancel, while the third term is always negative. This implies that the maximum of the prey nullcline gradually shifts to the left when cannibalism $(A)$ increases from 0 . In conclusion, the prey nullclines depend continuously upon $A$ as sketched in Fig.2a.

The nontrivial predator nullcline $d y / d t=0$ for model (7) (see Fig.2b) is the straight line

$$
A(1-E+d H) y=a(e-d h) x-d
$$

which intersects the $x$-axis at a point $x^{* *}$ independent upon $A$, namely

$$
x^{* *}=\frac{d}{a(e-d h)}
$$

and has a positive slope for all values of $A$ (recall that $E<1$ and $e>d h$ ).

Proof of property (i)

A direct consequence of condition (5) is that (see (9) and (10))

$$
x^{* *}>x^{*}
$$




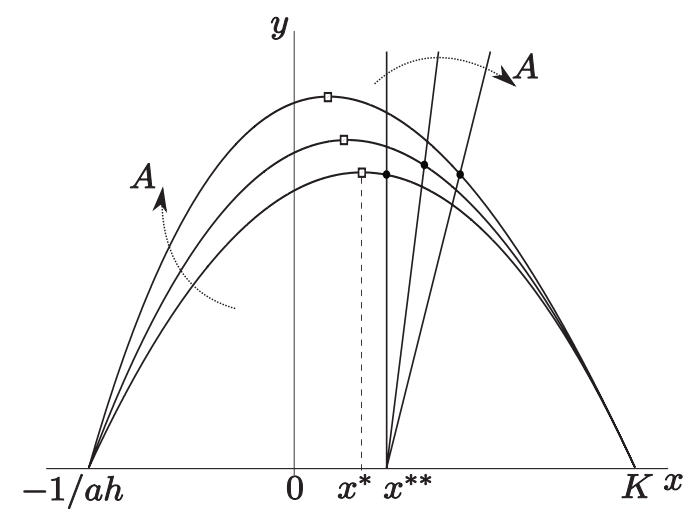

Figure 3: Prey and predator nullclines for different values of cannibalism $(A=0,0.9,1.8)$ when condition $(5)$ is satisfied : the equilibrium $(\bullet)$ is always on the right of the maximum of the prey nullcline $(\square)$. The curves are obtained with the same set of parameters used in Fig.2 except for $a=1.5$.

so that the nullclines are like in Fig.3, which clearly shows that the slopes of prey and predator nullclines are, respectively, negative and positive at the equilibrium point. But in prey-predator systems, where for biological reasons $J_{12}<0$ and $J_{21}>0$ (see eq.(7)), the negativity of the slope of the prey nullcline is equivalent to $J_{11}<0$, while the positivity of the slope of the predator isocline is equivalent to $J_{22}<0$. Thus, for all values of $A$ the signs of the four elements of the Jacobian matrix evaluated at the equilibrium are as follows

$$
J=\left[\begin{array}{ll}
- & - \\
+ & -
\end{array}\right]
$$

so that there is no activator and, hence, no Turing instability.

\section{Proof of property (ii)}

Condition (6) implies

$$
x^{* *}<x^{*}
$$

so that the nullclines are like in Fig.4. For $A=0$ model (7) is the Rosenzweig-MacArthur model (4) which has $J_{11}>0$ and $J_{22}=0$ since the trace of $J$ is positive (recall that the 
equilibrium is unstable). Increasing $A$ from zero, the two nullclines gradually vary and their slopes indicate that $J_{11}$ remains initially positive but decreases while $J_{22}$ becomes negative and decreases. Thus, the trace of $J$ decreases with $A$ until it becomes zero at a particular value, say $A_{1}$, of cannibalism (a Hopf bifurcation of model (7)). For $A$ slightly grater than $A_{1}$ the equilibrium is therefore stable and has

$$
J=\left[\begin{array}{ll}
+ & - \\
+ & -
\end{array}\right]
$$

so that the prey is an activator. A further increase of $A$, reduces $J_{11}$ until it becomes zero at $A=A_{2}$, i.e. when the predator nullcline intersects the prey nullcline at its maximum (where $J_{11}$ changes sign). Hence, for $A>A_{2}$

$$
J=\left[\begin{array}{ll}
- & - \\
+ & -
\end{array}\right]
$$

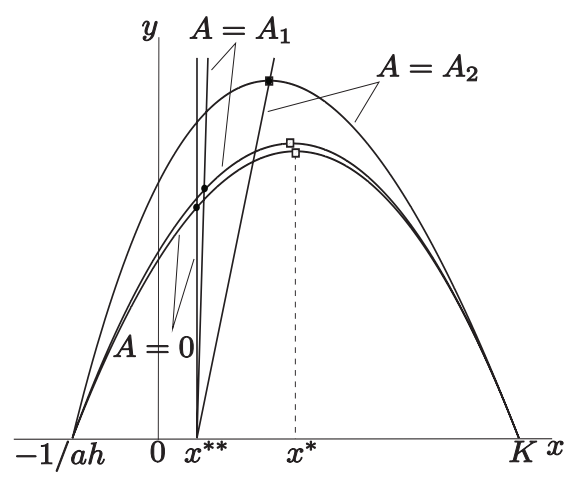

Figure 4: Prey and predator nullclines when condition (6) is satisfied. For $A=A_{1}$ model (7) has a Hopf bifurcation, while at $A=A_{2}$ (Turing bifurcation) the prey population ceases to be an activator. The curves are obtained with the same set of parameters used in Fig.2 except for $a=3.8$. The approximated critical values of cannibalism $(A)$ are $A_{1}=0.81211$ and $A_{2}=5.727616$. 
and again there is no activator. Thus, in conclusion, the equilibrium is stable and has the prey as activator for

$$
A_{1}<A<A_{2}
$$

so that spatial pattern can emerge provided cannibalism is sufficiently high (in agreement with (11)) and predator disperse much more than prey.

\section{Conclusions}

We have studied in this paper the relationship between cannibalism and pattern formation in spatially extended prey-predator systems, a subject investigated until now only in a paper published in this journal [20]. Our analysis is complete and based on a biologically sound model [9] and the proofs are presented in terms of prey and predator nullclines in order to facilitate ecologists who are mostly used to this technique.

The results are quite simple: spatial pattern can not emerge if, in the absence of cannibalism, the attractor of the model is an equilibrium, while they can emerge if, in the absence of cannibalism, the attractor is a limit cycle and predator cannibalism and dispersal are sufficiently high.

Our conclusions are most likely valid for other mechanisms promoting pattern formation in spatially extended prey-predator systems. For example, predator interference has already been shown to promote spatial pattern $[3,1]$ but a more careful analysis shows that this can occur exactly under the conditions described in this paper, i.e. interference cannot promote Turing instability if it is too low and the attractor is not a limit cycle in the absence of interference. We believe that it would be worth investing some effort to identify other mechanisms promoting pattern formation under the same conditions pointed out in this study. 


\section{References}

[1] D. Alonso, F. Bartumeus, and J. Catalan. Mutual interference between predators can give rise to Turing spatial patterns. Ecology, 83(1):28-34, 2002.

[2] M. Banerjee. Self-replication of spatial patterns in a ratio-dependent predator-prey model. Mathematical and Computer Modelling, 51(1-2):44 - 52, 2010.

[3] F. Bartumeus, D. Alonso, and J. Catalan. Self-organized spatial structures in a ratiodependent predator-prey model. Physica A: Statistical Mechanics and its Applications, 295(1-2):53 - 57, 2001.

[4] M. Baurmann, T. Gross, and U. Feudel. Instabilities in spatially extended predator-prey systems: Spatio-temporal patterns in the neighborhood of Turing-Hopf bifurcations. Journal of Theoretical Biology, 245(2):220 - 229, 2007.

[5] J. Beddington. Mutual interference between parasites or predators. Journal of Animal Ecology, 44:331-340, 1975.

[6] A. Chakraborty, M. Singh, D. Lucy, and P. Ridland. A numerical study of the formation of spatial patterns in twospotted spider mites. Mathematical and Computer Modelling, 49(9-10):1905-1919, 1996.

[7] A. De Roos, J. Metz, E. Evers, and A. Leipoldt. A size dependent predator-prey interaction: Who pursues whom? Journal of Mathematical Biology, 28(6):609-643, 1990.

[8] F. Della Rossa, S. Fasani, and S. Rinaldi. Potential Turing instability and application to plant-insect models. Submitted, 2011.

[9] F. Dercole and S. Rinaldi. Evolution of cannibalistic traits: Scenarios derived from adaptive dynamics. Theoretical Population Biology, 62(4):365 - 374, 2002. 
[10] M. Elgar and B. Crespi (eds.). Cannibalism: Ecology and Evolution among Diverse Taxa. Oxford University Press, 1992.

[11] G. Huxel and K. McCann. Food web stability: The influence of trophic flows across habitats. The American Naturalist, 152(3):460 - 469, 1998.

[12] S. Levin and L. Segel. Hypothesis for origin of planktonic patchiness. Nature, 259(5545):659, 1976.

[13] S. Lima. Strong preferences for apparently dangerous habitats - a consequence of differential escape from predators. OIKOS, 64(3):597-600, 1992.

[14] J. McNair. The effects of refuges on predator-prey interactions: A reconsideration. Theoretical Population Biology, 29(1):38-63, 1986.

[15] S. Rinaldi, S. Muratori, and Y. Kuznetsov. Multiple attractors, catastrophes and chaos in seasonally perturbed predator prey communities. Bulletin of Mathematical Biology, 55(1):15-35, 1993.

[16] M. Rosenzweig and R. MacArthur. Graphic representation and stability conditions of predator-prey interaction. The American Naturalist, 97:209 - 223, 1963.

[17] G. Ruxton. Short term refuge use and stability of predator-prey models. Theoretical Population Biology, 47(1):1 - 17, 1995.

[18] G. Ruxton, W. Gurney, and A. De Roos. Interference and generation cycles. Theoretical Population Biology, 42(3):235-253, 1992.

[19] R. Satnoianu, M. Menzinger, and P. Maini. Turing instabilities in general systems. Journal of Mathematical Biology, 41(6):493-512, 2000.

[20] G. Sun, G. Zhang, and Z. Jin. Predator cannibalism can give rise to regular spatial pattern in a predator-prey system. Nonlinear Dynamics, 58(1):75-84, 2009. 
[21] A. Turing. The chemical basis of morphogenesis. Philosophical Transactions of the Royal Society of London. Series B, Biological Sciences, 237(641):37-72, 1952.

[22] W. Wang, Q.-X. Liu, and Z. Jin. Spatiotemporal complexity of a ratio-dependent predator-prey system. Phys. Rev. E, 75(5):051913, 2007.

[23] L. Zhang, W. Wang, and Y. Xue. Spatiotemporal complexity of a predator-prey system with constant harvest rate. Chaos, Solitons and Fractals, 41(1):38 - 46, 2009. 